\title{
Analyse Multiniveau des Facteurs Associés au Retard dans la Vaccination des Enfants au Burkina Faso et au Mali
}

\author{
Ladji Binoko Sidibé1a ${ }^{\text {, Abdramane Soura }}{ }^{b}$ \\ a Direction Nationale de la Population, Bamako, Mali \\ b Maître Assistant, Institut Supérieur des Sciences de la Population, Ouagadougou, Burkina Faso
}

\begin{abstract}
Several studies conducted in developed countries and African Anglophone countries demonstrated that the context influences child immunisation status and the administration of specific vaccines. Nonetheless, few studies have focused on African Francophone countries, particularly the Sahel countries such as Burkina Faso and Mali. Analysing data from the Demographic and Health Surveys of Burkina Faso (2010) and Mali (2006) through a multilevel logistic regression approach, this article examines hypotheses stating that context influences the full and timely immunization of children aged 12 to 59 months even though individual characteristics have a larger effect. In richer enumeration sections in Mali, the children are less likely to receive a vaccine out of schedule. These results suggest that actors of health sector (particularly those intervening in national immunisation programs) should implement community approaches targeting local collective barriers to achieve full and timely vaccination of children in these countries.
\end{abstract}

Keywords: Childhood vaccination, immunisation delay, risk factors, DHS data, contextual effects, Burkina Faso, Mali

\section{Résumé}

Plusieurs études effectuées dans les pays développés et les pays anglophones d'Afrique montrent que le contexte influence le statut vaccinal des enfants ainsi que l'administration de certains vaccins spécifiques. Cependant, peu d'études ont porté spécifiquement sur les pays d'Afrique francophone, particulièrement ceux du Sahel comme le Burkina Faso et le Mali. En analysant les données provenant des enquêtes démographiques et de santé du Burkina Faso (2010) et du Mali (2006) à travers une approche de régression logistique multiniveau, cet article évalue dans quelle mesure le contexte influence le respect du calendrier vaccinal des enfants de 12 à 59 mois. Les résultats montrent que les variances contextuelles demeurent significatives dans le modèle final même si le respect du calendrier vaccinal reste surtout tributaire des caractéristiques individuelles de l'enfant. Notamment, au Mali, plus le niveau de vie moyen du district de recensement augmente, plus le risque pour un enfant de recevoir un vaccin en retard diminue. Ces résultats invitent les acteurs de la santé publique à mettre en œuvre des approches communautaires ciblant les barrières locales qui empêchent une vaccination correcte des enfants dans ces deux pays.

Mots-clés: Vaccination de routine, retard de vaccination, facteurs de risque, Données EDS, effet contextuel, Burkina Faso, Mali

\footnotetext{
I Adresse de l'auteur : Ibsidibe@yahoo.fr
} 


\section{Introduction}

La vaccination est une composante majeure des soins de santé primaires destinés à l'enfant depuis la conférence d'Alma-Ata de 1978. Cela a été rappelé également par l'Initiative de Bamako de 1987, la Conférence internationale sur la population et le développement tenue au Caire en 1994 et la vaccination occupe également une place de choix dans les politiques actuelles de santé menées au Burkina Faso et au Mali. Avant la conférence d'AlmaAta, les interventions en matière de vaccination visaient principalement l'éradication de la variole (Saliou, 2007). L'atteinte de cet objectif en 1977 a permis à l'Organisation mondiale de la santé de recommander un "élargissement des programmes de vaccination aux maladies que sont : la tuberculose, la poliomyélite, la diphtérie, le tétanos, la coqueluche et la rougeole; d'où la naissance des Programmes Elargis de Vaccination - PEV» (Saliou, 2007 ; p I 593). De nos jours, l'évolution de ces programmes a favorisé la prise en compte de nouveaux vaccins contre la fièvre jaune, l'hépatite virale $B(\mathrm{HepB})$, les méningites et les pneumonies dues à l'hémophilus influenzae B (Hib).

Des programmes nationaux de vaccination sont mis en œuvre par le Mali et le Burkina Faso depuis la fin de la décennie 70. Cependant, ces programmes tardent à montrer tout le bienfait qui en est attendu sur la baisse de la mortalité des enfants pour diverses raisons. Malgré sa tendance à la baisse depuis les années 1960, le niveau de mortalité infanto-juvénile reste encore très élevé dans les deux pays comparativement à la moyenne africaine (Masquelier, Reniers \& Pison, 2014) (Garenne et Gakusi, 2005). L'une des raisons citées est la faible vaccination des enfants contre les maladies évitables. En effet, non seulement la couverture vaccinale reste faible (CPS/MS et al., 2007 ; INSD et ICF International, 20I2) mais également une forte proportion d'enfants n'est pas vaccinée aux âges indiqués (Clark et Sanderson, 2009). Or, la combinaison de ces deux facteurs constituent en soi un risque épidémique majeur (Papania et Rodewald, 2006).

La couverture vaccinale complète est faible au Burkina Faso, et plus encore au Mali, comparativement aux autres pays d'Afrique de l'Ouest. Selon les données des enquêtes démographiques et de santé (EDS), respectivement $81,3 \%(2010)$ et $48,2 \%$ (2006) des enfants burkinabé et maliens de 12 à 23 mois ont reçu l'ensemble des vaccins de routine recommandés avant le premier anniversaire (CPS/MS et al., 2007 ; INSD et ICF International, 20I2). Cette faible couverture vaccinale des enfants est accompagnée d'un retard avéré aux âges recommandés de vaccination, de façon presque systématique au Mali (Clark et Sanderson, 2009). En outre, ce niveau de couverture vaccinale et de retard aux âges de vaccination cachent d'énormes disparités entre les différentes couches socioéconomiques, démographiques et culturelles de la population des deux pays.

Selon les données EDS, en 2010 au Burkina Faso, $73,3 \%$ des enfants âgés de 12-23 mois et vivant dans des ménages très pauvres étaient complètement vaccinés contre $83,9 \%$ des enfants vivant dans les ménages les plus nantis (INSD et ICF International, 20I2). Au Mali, ces écarts étaient respectivement de $48,6 \%$ contre $56,2 \%$ en 2006 (CPS/MS et al., 2006). A l'échelle régionale du Burkina Faso, la couverture vaccinale complète varie de $65,6 \%$ dans la région du Sahel à 93,8\% dans la région du Centre-Nord (INSD et ICF International, 20I2). Au Mali, elle varie de $28,7 \%$ dans la région de Kayes à $59,2 \%$ dans la capitale Bamako (CPS/MS et al., 2006). Par rapport au respect du calendrier vaccinal, Clark et Sanderson (2009) constataient que le Tchad, le Cambodge, le Mali, la Mauritanie et le Niger enregistrent systématiquement un retard important dans la vaccination des enfants aux âges recommandés.

Le Burkina Faso et le Mali ont également été choisis pour cette étude de cas sur la base de leur proximité géographique, culturelle, sanitaire et économique. En effet, ces deux pays partageant plusieurs centaines de kilomètres de frontière, et plusieurs groupes ethniques tels que les peulhs, les bobos, les touaregs et les markas. Paradoxalement, ces pays diffèrent toutefois en matière d'impact des programmes de vaccination puisque la couverture vaccinale complète chez les enfants de 12-23 mois varie du simple au double entre le Mali et le Burkina Faso (CPS/MS et al., 2007 ; INSD et ICF International, 20I2). Dans ces deux pays, on peut aussi déplorer la faiblesse de la production scientifique relative à la vaccination depuis l'instauration des programmes PEV en 1974. Selon Wiysonge et al. (20/3), il n'a été publié dans PubMed que 40 articles sur la vaccination des enfants au Burkina Faso et seulement 25 au Mali après 40 ans de mise en œuvre. Dans ce contexte, une nouvelle étude comparative du retard dans la vaccination des enfants devrait permettre de proposer des réorientations stratégiques en matière de vaccination en fonction des leçons apprises dans l'un ou l'autre pays.

\section{Considérations théoriques \\ Le calendrier vaccinal}

Le calendrier vaccinal tel que proposé par l'Organisation mondiale de la santé (OMS) et en vigueur au Burkina Faso et au Mali est celui illustré 
par la Figure I. II recense les vaccins dits de routine et recommande que tous les enfants reçoivent l'antigène :

- BCG et Polio 0, utilisés respectivement contre la tuberculose et la poliomyélite, dans les deux premières semaines qui suivent la naissance ;
- DTC et Polio comportant trois doses I, 2 et 3 utilisés contre la diphtérie, le tétanos, la coqueluche et la poliomyélite avant respectivement 6,10 et 14 semaines d'âges de l'enfant ;

- Rougeole et fièvre jaune, utilisés contre les maladies des mêmes noms dans un délai de 9 mois soit 39 semaines après la naissance.

Figure I : Calendrier vaccinal du Programme Elargi de Vaccination (Burkina Faso et Mali)

Source: Adapté de l'OMS, 2014
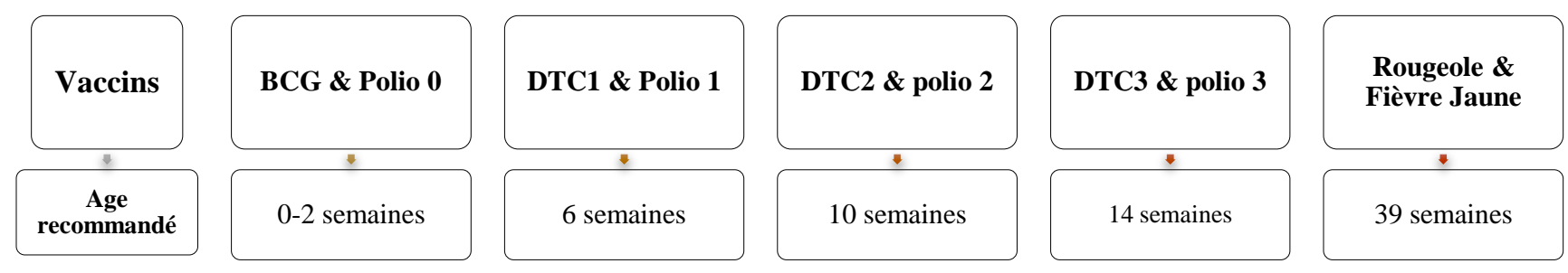

Fortement centré sur la petite enfance (moins de 9 mois d'âge), ce calendrier vaccinal fait l'hypothèse que «les agents pathogènes circulant dans la nature puissent entraîner des rappels naturels pour une protection de longue durée (Saliou, 2007). "Simplifié à la figure $\mathrm{I}$, le calendrier vaccinal $\left.\right|^{2}$ actuel enregistre continuellement l'intégration de nouveaux vaccins comme les «Penta I, 2 et 3 » comprenant les vaccins DTC/Hep/Hib+VPO en lieu et place des vaccins DTC et Polio I, 2 et 3.

\section{La notion de contexte ou de communauté}

Notre recherche vise à évaluer l'effet du "contexte" sur la vaccination des enfants et le respect du calendrier vaccinal. II convient donc de préciser ce concept clé. Le contexte est considéré comme un déterminant majeur de la santé en générale et particulièrement de celui des enfants (Diez-Roux et Mair, 2010; Pickett et Pearl, 200I). Dès les années 1990, Turrell (1995) insistait sur le fait que le contexte social, économique, physique et environnemental exerce un effet spécifique sur la santé des individus indépendamment des effets liés aux individus eux-mêmes.

Selon Pickett et Pearl (200I), les études touchant au contexte l'ont défini et mesuré différemment. A titre illustratif, le tableau I résume deux études s'intéressant au contexte au Burkina Faso (Sia et al., 2007) et au Nigeria (Babalola, 2008). Cet effet contextuel a été jugé significatif dans l'explication de

\footnotetext{
2 Pour plus d'informations sur le calendrier vaccinal ainsi que les maladies évitables par la vaccination, se référer à (USAID, 2006) sur le site http://www.usaid.gov/our_work/global_health.
}

la complétude vaccinale et des retards aux âges spécifiques de vaccinations dans les deux cas.

II ressort de ce tableau que la définition du contexte varie selon les études et il est paramétré dépendamment des objectifs de recherche. Sia et al. (2007) ont décrit le contexte à l'aide de quatre variables mesurées à ce niveau, tandis que Babalola (2008) n'en a utilisé pour caractériser le contexte. Ces auteurs ont tous imposé des effets aléatoires au contexte dans leurs modèles respectifs pour mettre en exergue l'effet contextuel (cf. Tableau I) .

Dans le cadre de cette étude, le contexte fait référence à une aire géographique permettant de caractériser l'environnement immédiat de l'individu. II est représenté par la zone de dénombrement (ZD). Les enquêtes démographiques et de santé utilisent ces découpages statistiques relativement réduits provenant du recensement général de la population et de l'habitat (RGPH). Les ZD permettent une collecte efficiente des données. Dans le cas du RGPH-2009 du Mali, la ZD également appelée section d'énumération (SE) était déterminée sur la base d'une population égale à 1100 habitants environ en milieu urbain et 700 habitants environ en milieu rural (INSTAT, 20I I).

Le choix des ZD comme contexte dans le cadre de cette étude se justifie par le fait que ces zones de dénombrement de taille réduite sont plus ou moins homogènes. Ainsi, il est possible de calculer des indicateurs au niveau du contexte (ici des moyennes statistiques). En effet, à vouloir trop étendre le contexte, le risque devient important que les moyennes ne le représentent plus (Pickett et Pearl, 200I). 
Tableau I : Modèle multiniveau et mesure du contexte dans deux travaux récents relatifs à la vaccination au Burkina Faso et au Nigeria

\begin{tabular}{|c|c|c|c|}
\hline Référence & & Sia et al., 2007 & Babalola, 2008 \\
\hline $\begin{array}{l}\text { Définition } \\
\text { contexte }\end{array}$ & du & District sanitaire & $\begin{array}{l}\text { Local government area (correspondant à la } \\
\text { municipalité au Burkina Faso et au Cercle au } \\
\text { Mali) }\end{array}$ \\
\hline $\begin{array}{l}\text { Indicateur } \quad d \\
\text { mesure } \\
\text { contexte et effet }\end{array}$ & $\begin{array}{l}\text { de } \\
\text { du }\end{array}$ & $\begin{array}{ll}\text { - } & \text { Pourcentage de femmes éduquées } \\
\text { - } & \text { Ratio population / personnel vaccinateur } \\
\text { - } & \text { Rayon moyen d'action des CSPS } \\
\text { - } & \text { Ratio habitants par CSPS } \\
\end{array}$ & Aucun \\
\hline Modèle d'analyse & & $\begin{array}{l}\text { Modèle logit multiniveau à deux niveaux: enfant et famille } \\
\text { (niveau I), district sanitaire (niveau 2) }\end{array}$ & $\begin{array}{l}\text { Modèle logit multiniveau à trois niveaux: enfant } \\
\text { (niveau I); famille (niveau 2) et communauté } \\
\text { (niveau 3) }\end{array}$ \\
\hline
\end{tabular}

Cet article vise à comprendre le phénomène du retard de vaccination des enfants en apportant des réponses à la question suivante: "Existe-t-il un lien entre les caractéristiques contextuelles et une vaccination tardive des enfants de 12 à 59 mois ?" Plus spécifiquement, il s'agit d'identifier les facteurs individuels et contextuels associées au retard de vaccination des enfants de 12 à 59 mois au Mali et au Burkina Faso et de déterminer la contribution du contexte dans l'explication. Pour ce faire, nous adoptons les hypothèses suivantes:

Hypothèse $[\mathrm{HI}]$ : Les caractéristiques contextuelles influencent le respect de son calendrier vaccinal indépendamment des caractéristiques individuelles.

Hypothèse [H2]: Malgré l'influence des caractéristiques contextuelles, le respect du calendrier vaccinal des enfants dépend davantage des caractéristiques individuelles associées à l'enfant, sa mère et son ménage.

La première hypothèse $[\mathrm{HI}]$ s'appuie sur plusieurs travaux de recherches sociologiques et épidémiologiques montrant que les seuls facteurs individuels ne peuvent expliquer l'ensemble des comportements de santé des individus et qu'une partie, aussi infime soit elle, est expliquée par les caractéristiques liées au contexte (Diez-Roux et Mair, 2010) (Turrell, 1995). Les auteurs s'accordent pour dire que les processus d'observation et d'imitation constituent l'un des canaux par lequel le contexte agit sur la santé des individus. Le voisinage est alors présenté comme un environnement favorable à l'apprentissage et aux échanges de procédés. Ce phénomène a été nommé par certains auteurs comme relevant de la «contagion sociale " (Galster, 2010). La littérature regorge également d'autres mécanismes d'influence comme les réseaux sociaux, la compétition sociale, l'environnement physique et institutionnel, etc.

Notre seconde hypothèse, qui suppose que les facteurs individuels restent prédominants dans l'explication des comportements de santé (hypothèse $[\mathrm{H} 2])$, provient du fait que la résolution des problèmes de santé est d'abord une affaire individuelle. Elle est fortement dépendante des caractéristiques de la personne et de sa famille (âge, sexe, taille du ménage, niveau de vie, etc.). Notamment, bien que l'offre de soins soit plus élevée en ville, les citadins ont des comportements de santé différents selon leurs capacités financières, leur niveau d'instruction, etc. II convient de noter à cet égard que les travaux parcourus dans le cadre de cette recherche identifient tous des effets contextuels faibles, comparativement aux effets individuels (Babalola, 2008 ; Sia et al., 2007 ; Wiysonge et al., 2012).

\section{Données et méthodes}

\section{Pays et échantillon d'étude}

Situés au cœur de l'Afrique de l'Ouest, à la lisière du Sahara, le Burkina Faso et le Mali demeurent à la traîne sur le plan du développement humain. Selon le rapport 2013 du Programme des nations unies pour le développement (PNUD, 20I3), ils étaient respectivement classés aux $183^{\mathrm{e}}$ et $182^{\mathrm{e}}$ rangs par rapport à l'indice du développement humain sur un total de 186 pays classés.

L'échantillon retenu dans cette étude comprend exclusivement les enfants âgés de 12 à 59 mois disposant d'un carnet de vaccination complètement rempli, permettant la détermination des âges de vaccination. Ce choix d'échantillon s'impose puisque les âges de vaccination, exprimés en semaines, ne peuvent être calculés pour la plupart des enfants ne disposant pas de carnet de vaccination car les jours et mois de vaccination, nécessaires au calcul, sont manquants. Au total, l'échantillon comprend 3116 enfants au Burkina Faso et 2180 enfants au Mali (après pondération). Au Mali, l'échantillon d'enfants inclus dans les analyses représentent $53 \%$ des enfants ayant un carnet de vaccination et $44 \%$ au Burkina Faso.

Il faut évidemment reconnaître que ce choix peut entrainer des biais dans la mesure réelle du 
phénomène. En effet, les enfants de 12-23 mois ne disposant pas de carnet de vaccination constituent près de $39,2 \%$ des enfants enquêtés au Mali et $16,7 \%$ de ceux enquêtés au Burkina Faso. Ils sont en général moins vaccinés que les autres enfants, ou plus tardivement (CPS/MS et al., 2007 ; INSD et ICF International, 20I2).

\section{Données et variables}

Les données de cette étude proviennent des enquêtes démographiques et de santé (EDS) réalisées par le Burkina Faso en 2010 et le Mali en 2006. Compte tenu de la thématique abordée, l'intérêt a porté sur la section du questionnaire portant sur la vaccination des enfants en plus des caractéristiques de la femme, celles du ménage et celles de la zone de dénombrement. Dans la section "vaccination des enfants » du questionnaire, les informations étaient collectées sur les enfants vivant de moins de 5 ans au moment de l'enquête soit à partir du carnet de vaccination soit à partir des déclarations de la mère (CPS/MS et al., 2007 ; INSD et ICF International, 20I2).

Le retard aux âges spécifiques de vaccination : Selon le calendrier vaccinal (cf. figure I), chaque vaccin devrait être administré à un âge spécifique. La mesure du retard proposée par l'étude tient compte de cet aspect et maintient un délai maximal de quatre semaines à l'âge recommandé afin que le vaccin puisse être considéré comme administré à temps. Cela a été fait en considérant les vaccins de routines que sont : le BCG, les trois doses de DTC et de polio et celui contre la rougeole. II traduit la notion de statut vaccinal complet et correct de l'enfant. Ainsi, tout enfant qui reçoit à la fois les vaccins du calendrier vaccinal simplifié (figure I) en respectant les délais suivants: BCG avant 6 semaines d'âge; $D T C I+$ Poliol avant 10 semaines d'âge; DTC2+Polio2 avant 14 semaines d'âge; DTC3+Polio3 avant 18 semaines d'âge et Rougeole avant 43 semaines d'âge est déclaré avoir un statut vaccinal complet et correct. Ces enfants sont codés 0 . Dans le cas contraire, le statut vaccinal est déclaré incorrect et codé I. Ainsi, les enfants ayant reçu quelques vaccins seulement avant de compléter leur premier anniversaire sont d'office en retard vaccinal. Egalement, cette définition permet qu'un enfant ait un statut vaccinal complet mais incorrect si un ou plusieurs vaccins n'ont pas été administrés aux âges recommandés.

Tirées de la littérature, les variables explicatives sont relatives à l'enfant, sa mère, sa famille et au contexte de résidence de la famille i.e. la zone de dénombrement. Plus spécifiquement, celles retenues dans l'étude au niveau individuel sont: le sexe de l'enfant, son groupe d'âge, son rang de naissance, son lieu de naissance et l'âge de sa mère à la naissance, le niveau d'instruction de la mère, sa religion, son statut matrimonial, son groupe ethnique et le niveau de vie de son ménage (un proxy construit à partir de la possession de biens par le ménage et fournis avec la base de données). Les variables retenues au niveau contextuel sont: la région et les niveaux d'instruction et niveaux de vie "contextuels», qui sont des agrégats au niveau de la zone de dénombrement.

\section{Méthodes statistiques}

En plus de l'analyse descriptive, cette recherche se base principalement sur l'analyse logistique multiniveau. L'analyse descriptive servira à décrire le retard vaccinal tandis que l'analyse logistique multiniveau permet de tester l'influence du voisinage dans l'explication du phénomène. Les modèles sont estimés à travers les logiciels MLwiN 2.30 et STATA 12. Leckie et Charlton (2012) (Université de Bristol, Grande Bretagne) ont décrit un processus à partir de la commande "runmlwin » permettant de spécifier un modèle multiniveau sous STATA, de l'exécuter directement sous $M L w i N$ et de récupérer les résultats sous STATA.

Les modèles ont été estimés pas à pas en trois étapes, correspondant à la spécification de trois modèles pour chacun des pays. II s'agit du modèle vide (M0) ne comportant aucune variable explicative, du modèle (MI) intégrant les variables individuelles et du modèle final (M2) faisant intervenir les variables contextuelles en plus des variables individuelles. Cette démarche de spécification du modèle à l'avantage de rendre compte de l'effet de l'addition des variables d'un niveau hiérarchiquement supérieur sur le modèle du niveau précédent et de constater le renforcement ou l'atténuation de l'effet d'un groupe de facteurs. L'équation de régression s'écrit comme suit :

$$
\begin{aligned}
& Y_{i j} \rightarrow \operatorname{Bernouilli}\left(\pi_{i j}\right) \text { et } \\
& \operatorname{Logit}\left(\pi_{i j}\right)=\operatorname{Ln}\left(\frac{\pi_{i j}}{1-\pi_{i j}}\right)=\beta_{0 j} \operatorname{cons}+\sum_{j=1}^{k} \beta_{j} X_{i j}+\sum_{j}^{k} \alpha_{j} T_{i j} \\
& \text { où } \beta_{0 j}=\beta_{0}+\mathrm{u}_{0 \mathrm{j}} \text { et } \mathrm{u}_{0 \mathrm{j}} \rightarrow \mathrm{N}\left(0, \sigma_{\mathrm{u}_{0}}^{2}\right) \\
& \quad \text { où } X_{i j} \text { fait référence à la variable individuelle } X
\end{aligned}
$$
observée sur l'enfant $i$ appartenant à la zone de dénombrement ${ }^{j}$. De même, $T_{i j}$ fait référence à la variable contextuelle $T$ observée sur l'enfant $i$ appartenant à la zone de dénombrement $j$.

Un accent particulier est mis sur l'examen du coefficient de partition de la variance (CPV) qui mesure la proportion de la variance totale qui réside au niveau du contexte. Dans le modèle à deux niveaux, il correspond également au coefficient de 
corrélation intra-classe qui mesure la ressemblance entre deux enfants de la même zone de dénombrement (Leckie et Charlton, 2012). Une faible valeur du CPV indique clairement que le risque pour un enfant de recevoir le vaccin en retard dépend davantage des différences individuelles que des différences entre les zones de dénombrement. Le modèle multiniveau apporte donc plus d'éclaircissement dans l'explication de la variation du retard de vaccination qu'un modèle classique. Par exemple, un CPV de $20 \%$ signifie qu'une cinquième de la variation dans les risques de ne pas se faire vacciner ou de se faire vacciner en retard réside dans les différences entre les zones de dénombrement, tandis que $80 \%$ de cette variation est expliqué par des différences individuelles. Une variance contextuelle significativement différente de 0 permet également de conclure que le contexte influence bien le retard de vaccination (Leckie et Charlton, 20I2).

\section{Résultats}

\section{Description de l'échantillon}

L'analyse de la composition de l'échantillon (cf. Tableau 2) permet de mettre en évidence certaines similitudes entre les deux pays. Par exemple, comme attendu les garçons sont légèrement plus nombreux que les filles, tant au Burkina Faso qu'au Mali. Au Mali, les garçons représentent $52,9 \%$ de l'échantillon contre $47,1 \%$ de filles. Ces chiffres sont respectivement de $52,7 \%$ et de $47,3 \%$ au Burkina Faso. Selon l'âge, environ le tiers de l'échantillon ( $31,3 \%$ au Burkina Faso et 36,5\% au Mali) est âgé de 12 à 23 mois et près de 7 enfants sur 10 sont nés de mères âgées de 20 à 34 ans au moment de la naissance dans les deux pays. On note également une proportion importante d'enfants dont la mère est sans instruction, soit $8 \mathrm{I}, \mathrm{I} \%$ au Burkina Faso contre $79,4 \%$ au Mali.

La composition ethnique (cf. Tableau 2) confirme que les mossis constituent le groupe ethnique majoritaire au Burkina Faso $(53,2 \%)$ tandis qu'au Mali, ce sont les bambaras qui dominent la structure ethnique de l'échantillon (37,2\%). Les peulhs, groupe ethnique dont l'activité principale est tournée vers l'élevage, existent dans les pays mais dans des proportions différentes (10,3\% au Mali contre 6,8\% au Burkina Faso si on y ajoute les touaregs).

Au-delà de ces quelques ressemblances, le Mali et le Burkina Faso se distinguent sur le plan de la fréquence de l'assistance à l'accouchement (cf. Tableau 2). En effet, une proportion plus importante d'enfants maliens (38,7\%) nait hors des formations sanitaires contre $24,6 \%$ des enfants burkinabé. Sur le plan religieux, la plupart des enfants maliens ont leur mère de confession musulmane $(96,4 \%$ contre 63,6\% des enfants burkinabé).

Finalement, au Burkina Faso, l'échantillon est dominé par les enfants provenant de la région de la Boucle du Mouhoun, de celle des Hauts Bassins et du Centre-Est qui représentent respectivement 14,3\%, $13,2 \%$ et $10,1 \%$ de l'échantillon. Au Mali, c'est la région de Sikasso qui compte le plus d'enfants (24,9\%). Les régions Nord de ce pays (Tombouctou, Gao et Kidal) représentent seulement $6,8 \%$ de l'échantillon.

Tableau 2 : Distribution de l'échantillon d'enfants selon les facteurs étudiés

\begin{tabular}{|c|c|c|c|c|}
\hline \multirow{2}{*}{ FACTEURS ETUDIES } & \multicolumn{2}{|c|}{ Burkina Faso (20I0) } & \multicolumn{2}{|c|}{ Mali (2006) } \\
\hline & Effectif & $\%$ & Effectif & $\%$ \\
\hline \multicolumn{5}{|l|}{ Sexe de l'enfant } \\
\hline Fille & I 474 & $47,3 \%$ & I 027 & $47,1 \%$ \\
\hline Garçon & 1642 & $52,7 \%$ & 1153 & $52,9 \%$ \\
\hline \multicolumn{5}{|l|}{ Age de l'enfant } \\
\hline $12-23$ mois & 976 & $31,3 \%$ & 796 & $36,5 \%$ \\
\hline 24-59 mois & 2140 & $68,7 \%$ & 1383 & $63,5 \%$ \\
\hline \multicolumn{5}{|l|}{ Rang de naissance } \\
\hline Rang I & 635 & $20,4 \%$ & 325 & $14,9 \%$ \\
\hline Rang 2-5 & I 775 & $57,0 \%$ & 1230 & $56,4 \%$ \\
\hline Rang 6 ou + & 706 & $22,6 \%$ & 625 & $28,7 \%$ \\
\hline \multicolumn{5}{|c|}{ Age de la mère à la naissance } \\
\hline$<20$ ans & 405 & $13,0 \%$ & 328 & $15,0 \%$ \\
\hline $20-34$ ans & 2199 & $70,6 \%$ & 1518 & $69,7 \%$ \\
\hline $35-49$ ans & 512 & $16,4 \%$ & 334 & $15,3 \%$ \\
\hline \multicolumn{5}{|l|}{ Lieu de naissance } \\
\hline Structure de santé & 2349 & $75,4 \%$ & 1337 & $61,3 \%$ \\
\hline Maison et autre lieu & 767 & $24,6 \%$ & 843 & $38,7 \%$ \\
\hline \multicolumn{5}{|l|}{ Niveau d'instruction } \\
\hline Aucun & 2526 & $81,1 \%$ & | 73| & $79,4 \%$ \\
\hline
\end{tabular}




\begin{tabular}{|c|c|c|c|c|c|}
\hline \multirow{2}{*}{ FACTEURS ETUDIES } & \multicolumn{2}{|c|}{ Burkina Faso (20।0) } & \multicolumn{3}{|l|}{ Mali (2006) } \\
\hline & Effectif & $\%$ & Effectif & & $\%$ \\
\hline Primaire & 407 & $13,1 \%$ & 267 & & $12,2 \%$ \\
\hline Secondaire ou + & 183 & $5,9 \%$ & 182 & & $8,4 \%$ \\
\hline Religion & & & Religion & & \\
\hline Chrétienne & 903 & $29,0 \%$ & Autre & 78 & $3,6 \%$ \\
\hline Musulmane & 1983 & $63,6 \%$ & Musulmane & 2102 & $96,4 \%$ \\
\hline Autre & 229 & $7,4 \%$ & & & \\
\hline \multicolumn{6}{|l|}{ Niveau de vie du ménage } \\
\hline Le plus Pauvre & 517 & $16,6 \%$ & 396 & & $18,2 \%$ \\
\hline Pauvre & 629 & $20,2 \%$ & 364 & & $16,7 \%$ \\
\hline Moyen & 709 & $22,8 \%$ & 394 & & $18,1 \%$ \\
\hline Riche & 705 & $22,6 \%$ & 453 & & $20,8 \%$ \\
\hline Le plus riche & 555 & $17,8 \%$ & 572 & & $26,3 \%$ \\
\hline \multicolumn{6}{|l|}{ Statut matrimonial } \\
\hline Mariée & 2945 & $94,5 \%$ & 2087 & & $95,7 \%$ \\
\hline Autre & $17 \mid$ & $5,5 \%$ & 93 & & $4,3 \%$ \\
\hline Ethnie & & & Ethnie & & \\
\hline Mossi & I 657 & $53,2 \%$ & Bambara & 686 & $31,5 \%$ \\
\hline Bobo, Dioula, Senoufo & 331 & $10,6 \%$ & Sénoufo, Minianka & 298 & $13,7 \%$ \\
\hline Peul, Touareg & 211 & $6,8 \%$ & Peulh & 225 & $10,3 \%$ \\
\hline Lobi, Dagara & 196 & $6,3 \%$ & Sarakolé & 254 & $11,6 \%$ \\
\hline Gourmatché & 156 & $5,0 \%$ & Sonrhaï, Tamasheq & 223 & $10,2 \%$ \\
\hline Gourounsi & 162 & $5,2 \%$ & Dogon & 198 & $9,1 \%$ \\
\hline Bissa & 183 & $5,9 \%$ & Malinké & 138 & $6,4 \%$ \\
\hline Autre & 219 & $7,0 \%$ & Autre & 158 & $7,2 \%$ \\
\hline Région & & & Région & & \\
\hline Centre & 334 & $8,3 \%$ & Bamako & 381 & $17,5 \%$ \\
\hline Boucle du Mouhoun & 445 & $14,3 \%$ & Kayes & 188 & $8,6 \%$ \\
\hline Cascades & 89 & $2,9 \%$ & Koulikoro & 349 & $16,0 \%$ \\
\hline Sahel & 142 & $4,5 \%$ & Sikasso & 543 & $24,9 \%$ \\
\hline Centre-Est & 315 & $10,1 \%$ & Ségou & 410 & $18,8 \%$ \\
\hline Centre-Nord & 286 & $9,2 \%$ & Mopti & 167 & $7,7 \%$ \\
\hline Centre-Ouest & $24 I$ & $7,7 \%$ & Tombouctou & 58 & $2,7 \%$ \\
\hline Centre-Sud & 174 & $5,6 \%$ & Gao/Kidal & 84 & $3,9 \%$ \\
\hline Est & 232 & $7,4 \%$ & - & & - \\
\hline Hauts Bassins & 410 & $13,2 \%$ & - & & - \\
\hline Nord & 257 & $8,2 \%$ & - & & - \\
\hline Plateau Central & 149 & $4,8 \%$ & - & & - \\
\hline Sud-Ouest & 118 & $3,8 \%$ & - & & - \\
\hline Ensemble & 3116 & $100,0 \%$ & 2180 & & $100,0 \%$ \\
\hline
\end{tabular}

Pour les variables contextuelles continues (niveau de vie contextuel et niveau d'instruction contextuel), le tableau 3 présente quelques statistiques descriptives. II ressort de l'analyse de ce tableau que la proportion moyenne de femmes instruites des zones de dénombrement est de $22 \%$ au Burkina Faso contre $18,1 \%$ au Mali. En comparant les moyennes du score factoriel moyen (niveau de vie) aux écart-types dans les deux pays, on peut mettre en évidence la grande variation du niveau de vie tant au Mali qu'au Burkina Faso entre les contextes. Cette variabilité est moindre quand on examine la proportion de femmes instruites. 
Tableau 3: Quelques statistiques descriptives sur les facteurs contextuels continus

\begin{tabular}{|c|c|c|c|c|c|}
\hline Facteurs contextuels & Effec & Minimum & Moyenne & Maximum & Ecart type \\
\hline \multicolumn{6}{|l|}{ Burkina Faso (20I0) } \\
\hline $\begin{array}{l}\text { Niveau de vie } \\
\text { ou score factoriel moyen }\end{array}$ & 572 & $-8,8864$ & 0,0365 & 46,6463 & 8,1050 \\
\hline $\begin{array}{l}\text { - Niveau d'instruction ou } \\
\text { proportion de femmes instruites }\end{array}$ & 572 & 0,0000 & 0,2199 & 1,0000 & 0,2433 \\
\hline \multicolumn{6}{|l|}{ Mali (2006) } \\
\hline $\begin{array}{l}\text { Niveau de vie } \\
\text { ou score factoriel moyen }\end{array}$ & 388 & $-9,9595$ & 1,2026 & 36,1160 & 9,2351 \\
\hline $\begin{array}{l}\text { - Niveau d'instruction ou } \\
\text { proportion de femmes instruites }\end{array}$ & 388 & 0,0000 & 0,1808 & 1,0000 & 0,2218 \\
\hline
\end{tabular}

\section{Analyse descriptive}

Malgré l'existence du calendrier vaccinal de l'enfant et la mise en œuvre d'efforts politiques pour son application, force est de constater que le calendrier n'est pas correctement respecté pour une part importante des enfants dans les deux pays. Le schéma vaccinal des enfants, tel que illustré par les graphes 1 et 2, montre une grande diversité des pratiques dans l'âge à la vaccination des enfants. Ces graphes présentent le nombre d'enfants vaccinés en fonction de leur âge en semaines à l'administration des vaccins: $B C G, D T C ~ I, 2,3$ et rougeole.

Le schéma vaccinal des enfants garde la même allure dans les deux pays (cf. graphes I et 2). Un pic de vaccination est enregistré aux alentours des âges recommandés de vaccination, traduisant un succès relatif des efforts déployés tant par les parents que par le système sanitaire pour faire respecter le calendrier vaccinal. Par contre, des fréquences encore élevées sont constatées aux âges éloignés avant et/ou après l'âge recommandé, mettant en évidence les situations qui ne respectent pas les âges recommandés à la vaccination.

Notons que le DTCl et le Polio I suivent le même schéma vaccinal, de même que le DTC2 et le Polio 2, le DTC3 et le Polio 3. C'est pourquoi les courbes représentant les vaccins que sont le polio I, 2 et 3 ne figurent pas sur les graphes $I$ et 2 .

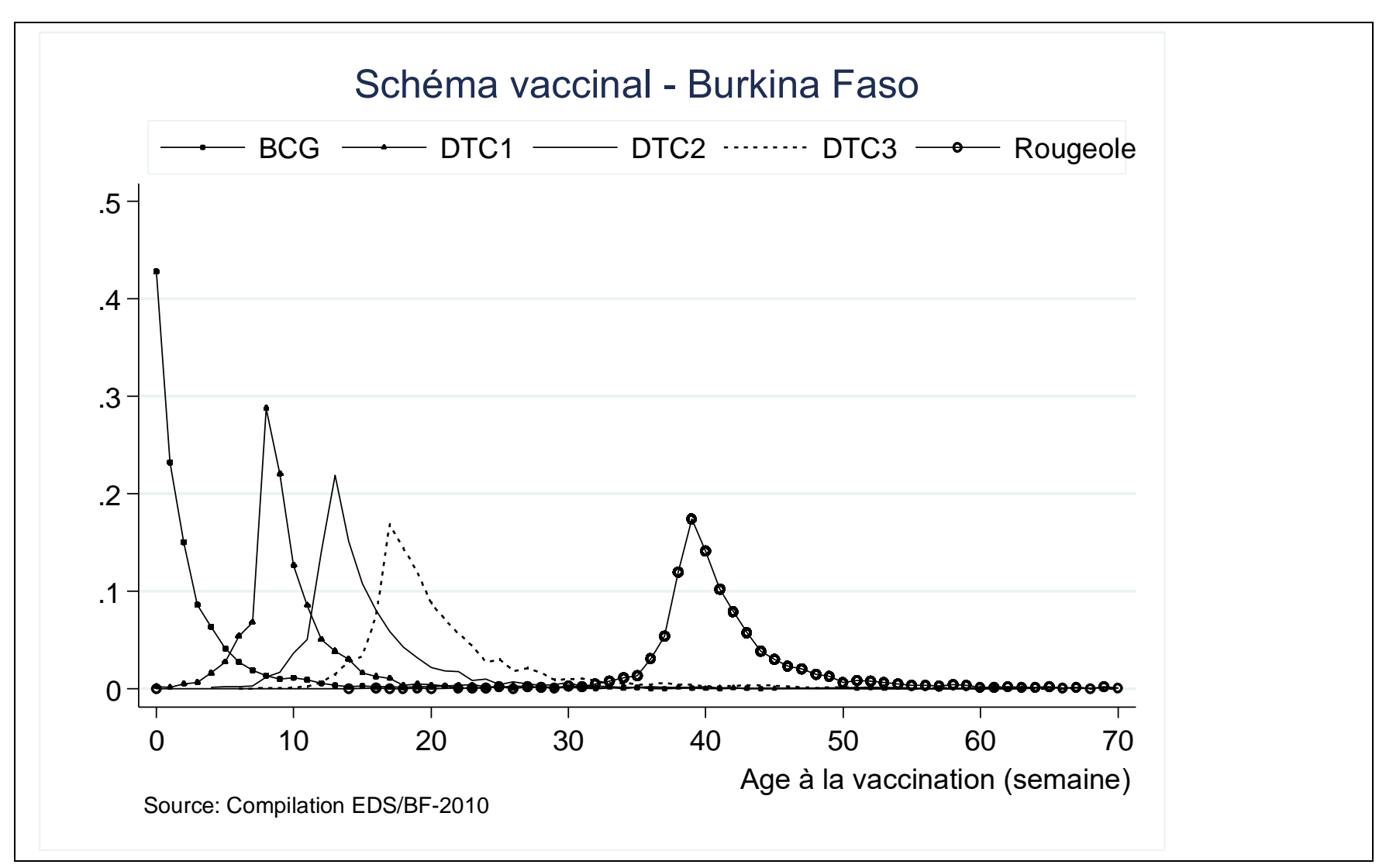

Figure 2 : Schéma vaccinal des enfants au Burkina Faso (2010) 


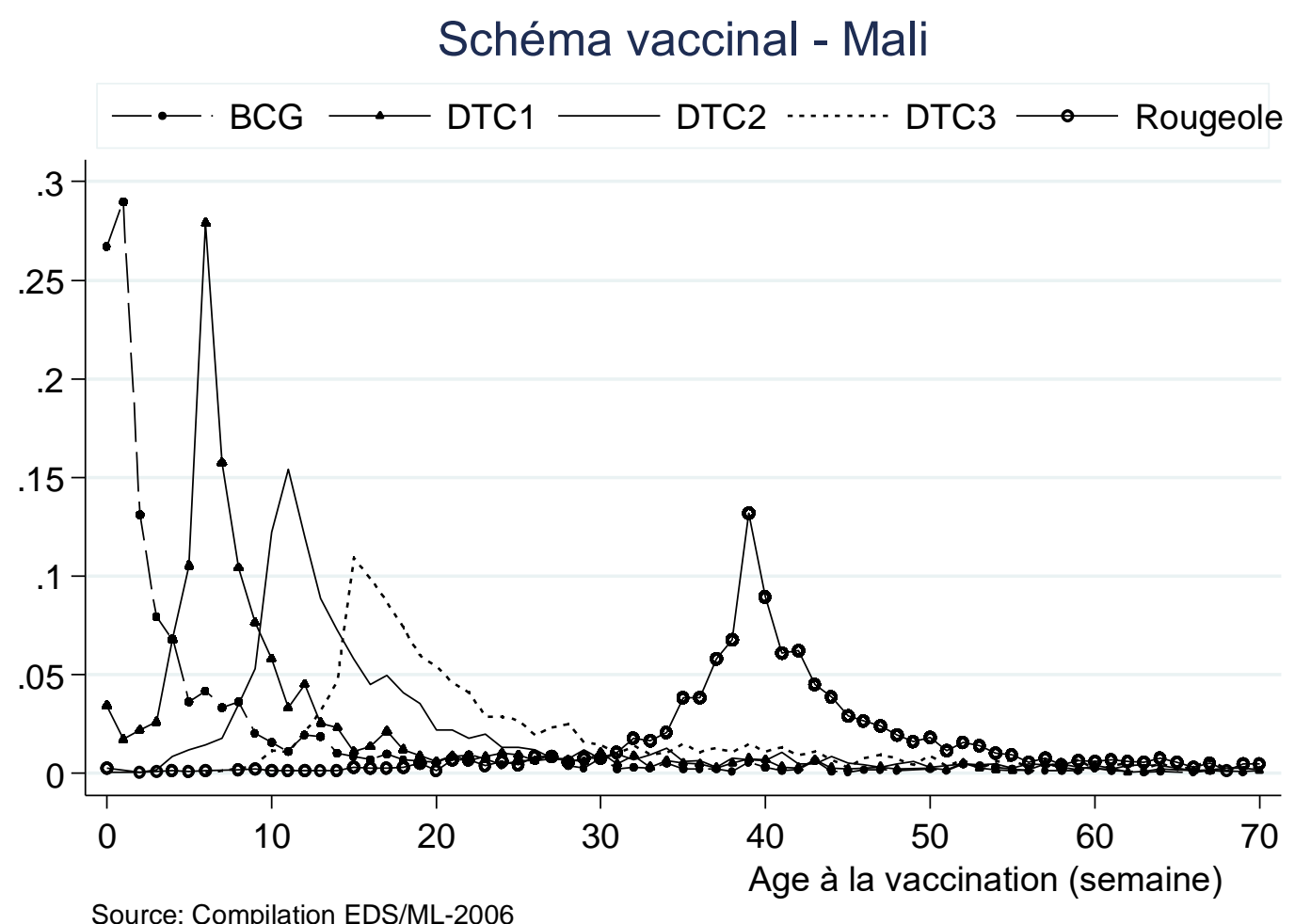

Source: Compilation EDS/ML-2006

Figure 3 : Schéma vaccinal des enfants au Mali (2006)

En portant l'attention sur le respect des âges spécifiques de vaccination, les chiffres contenus dans le tableau 4 montrent que le phénomène du retard est très important dans les deux pays puisqu'il touche un peu plus de 7 enfants sur 10. Respectivement $75,5 \%$ et $71,0 \%$ des enfants burkinabé et maliens disposant de carnet de vaccination enregistrent du retard sur la prise d'au moins un vaccin de routine.

Globalement, toutes les caractéristiques relatives à l'enfant présentent une relation statistiquement significative avec le retard de vaccination au Mali, excepté le sexe et le groupe d'âge de l'enfant. Plus le rang de naissance de l'enfant est élevé, plus la proportion d'enfants enregistrant du retard à la vaccination est élevée. Ainsi, la proportion d'enfants maliens ayant enregistré du retard aux âges spécifiques de vaccination est plus importante chez ceux de rang 6 ou plus $(74,2 \%)$ que chez ceux de rang I (64,9\%) (cf. Tableau 4). Au Burkina, 77,8\% des enfants de rang 6 ou plus et $71,8 \%$ de ceux de rang I enregistrent du retard à la vaccination. Nos résultats indiquent également que le fait de naître dans une formation sanitaire est significativement associé à une vaccination à temps.

Par rapport à la mère, toutes les caractéristiques retenues sont significativement associées au retard de vaccination dans l'un ou l'autre pays. Le retard de vaccination est notamment plus marqué chez les enfants dont la mère n'est pas instruite. Tandis que $60,1 \%$ des enfants burkinabé enregistrent du retard de vaccination lorsque leur mère est diplômée du secondaire (ou plus), $76,5 \%$ de ceux dont la mère n'est pas instruite enregistrent un retard (cf. Tableau 4). Ces chiffres sont respectivement de $47,3 \%$ et $74,2 \%$ au Mali. Dans ce pays, les enfants des mères non mariées semblent également être disproportionnellement vaccinés en retard. C'est le phénomène inverse qui est observé au Burkina Faso où $76,1 \%$ des enfants dont la mère est mariée enregistrent un retard de vaccination, contre $66,3 \%$ de ceux dont la mère ne l'est pas.

L'analyse de la relation entre les niveaux d'instruction et de vie contextuels et le retard de vaccination montre une association significative au Mali alors qu'au Burkina Faso ces relations ne le sont pas. Plus le niveau d'instruction contextuel est élevé, moins important est le retard de vaccination chez les enfants maliens. II en va de même pour le niveau de vie contextuel. L'analyse des différences régionales (cf. Tableau 4) montrent qu'au Burkina Faso le retard de vaccination est particulièrement important dans trois régions, à savoir le Sahel, le Nord et les Cascades. Au Mali, trois régions (Sikasso, Tombouctou et Koulikoro) sont à la traine par rapport au respect des âges de vaccination chez les enfants. 
Tableau 4 : Relation bivariée entre les variables explicatives et le retard de vaccination

\begin{tabular}{|c|c|c|c|c|c|}
\hline \multirow[b]{2}{*}{ FACTEURS ÉTUDIÉS } & \multicolumn{2}{|c|}{ Burkina Faso (20।0) } & \multicolumn{3}{|l|}{ Mali (2006) } \\
\hline & Effectif & $\begin{array}{l}\% \text { en retard de } \\
\text { vaccination }\end{array}$ & Effectif & & $\begin{array}{l}\% \text { en retard } \mathrm{de} \\
\text { vaccination }\end{array}$ \\
\hline Sexe de l'enfant & & ns & & & ns \\
\hline Fille & I 474 & $75,7 \%$ & I 027 & & $70,0 \%$ \\
\hline Garçon & 1642 & $75,3 \%$ & 1153 & & $71,8 \%$ \\
\hline Age de l'enfant & & ns & & & ns \\
\hline $12-23$ mois & 976 & $74,0 \%$ & 796 & & $72,0 \%$ \\
\hline $24-59$ mois & 2140 & $76,2 \%$ & I 384 & & $70,4 \%$ \\
\hline Rang de naissance & & 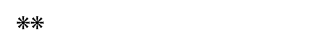 & & & *** \\
\hline Rang I & 635 & $71,8 \%$ & 325 & & $64,9 \%$ \\
\hline Rang 2-5 & I 776 & $75,9 \%$ & 1230 & & $70,9 \%$ \\
\hline Rang 6 ou + & 705 & $77,8 \%$ & 625 & & $74,2 \%$ \\
\hline Age de la mère à la naissance & & ns & & & *** \\
\hline$<20$ ans & 406 & $76,5 \%$ & 327 & & $69,4 \%$ \\
\hline $20-34$ ans & 2199 & $75,8 \%$ & 1519 & & $70,0 \%$ \\
\hline $35-49$ ans & 511 & $73,6 \%$ & 334 & & $77,2 \%$ \\
\hline Lieu de naissance & & **** & & & ***** \\
\hline Maison (ou autre) & 767 & $85,1 \%$ & 843 & & $82,0 \%$ \\
\hline Structure de santé & 2349 & $72,4 \%$ & I 337 & & $64,0 \%$ \\
\hline Niveau d'instruction & & **** & & & ***** \\
\hline Aucun & 2526 & $76,5 \%$ & 1730 & & $74,2 \%$ \\
\hline Primaire & 407 & $76,2 \%$ & 267 & & $66,3 \%$ \\
\hline Secondaire ou + & 183 & $60,1 \%$ & 183 & & $47,3 \%$ \\
\hline Niveau de vie du ménage & & *** & & & **** \\
\hline Le plus pauvre & 517 & $78,9 \%$ & 394 & & $80,2 \%$ \\
\hline Pauvre & 629 & $76,8 \%$ & 364 & & $81,6 \%$ \\
\hline Moyen & 709 & $76,7 \%$ & 396 & & $82,1 \%$ \\
\hline Riche & 706 & $75,6 \%$ & 454 & & $65,3 \%$ \\
\hline Le plus riche & 556 & $69,2 \%$ & 572 & & $54,7 \%$ \\
\hline Statut matrimonial & & **⿻丷木 & & & ns \\
\hline Mariée & 2945 & $76,1 \%$ & 2086 & & $70,8 \%$ \\
\hline Autre & $|7|$ & $66,3 \%$ & 94 & & $75,3 \%$ \\
\hline Religion & & **** & Religion & & $*$ \\
\hline Chrétienne & 903 & $69,9 \%$ & Autre & 79 & $79,7 \%$ \\
\hline Musulmane & 1983 & $77,7 \%$ & Musulmane & 2101 & $70,6 \%$ \\
\hline Autre & 230 & $78,6 \%$ & & & \\
\hline Ethnie & & *** & Ethnie & & ***** \\
\hline Mossi & 1657 & $74,9 \%$ & Bambara & 686 & $72,7 \%$ \\
\hline Bobo, Dioula, Senoufo & 331 & $78,9 \%$ & Sénoufo & 298 & $84,1 \%$ \\
\hline Peul, Touareg & 211 & $84,2 \%$ & Peulh & 225 & $74,4 \%$ \\
\hline Lobi, Dagara & 196 & $76,1 \%$ & Sarakolé & 254 & $72,5 \%$ \\
\hline Gourmatché & 156 & $72,7 \%$ & Sonrhaï & 223 & $64,7 \%$ \\
\hline Gourounsi & 162 & $67,3 \%$ & Dogon & 198 & $61,2 \%$ \\
\hline Bissa & 183 & $71,0 \%$ & Malinké & 138 & $66,4 \%$ \\
\hline Autre & 219 & $76,7 \%$ & Autre & 158 & $71,8 \%$ \\
\hline Région & & **** & Région & & ***** \\
\hline Centre & 258 & $73,6 \%$ & Bamako & 381 & $52,2 \%$ \\
\hline Boucle du Mouhoun & 444 & $74,8 \%$ & Kayes & 188 & $70,7 \%$ \\
\hline Cascades & 89 & $86,5 \%$ & Koulikoro & 348 & $77,4 \%$ \\
\hline Sahel & 142 & $88,0 \%$ & Sikasso & 543 & $84,9 \%$ \\
\hline Centre-Est & 315 & $67,3 \%$ & Ségou & 410 & $69,8 \%$ \\
\hline Centre-Nord & 285 & $76,2 \%$ & Mopti & 167 & $56,3 \%$ \\
\hline Centre-Ouest & 241 & $79,3 \%$ & Tombouctou & 58 & $84,5 \%$ \\
\hline Centre-Sud & 174 & $56,3 \%$ & Gao/Kidal & 84 & $65,5 \%$ \\
\hline Est & 232 & $72,4 \%$ & & & - \\
\hline Hauts Bassins & 410 & $79,0 \%$ & & & - \\
\hline Nord & 257 & $84,0 \%$ & & & - \\
\hline
\end{tabular}




\begin{tabular}{|c|c|c|c|c|}
\hline \multirow[b]{2}{*}{ FACTEURS ÉTUDIÉS } & \multicolumn{2}{|c|}{ Burkina Faso (20I0) } & \multicolumn{2}{|l|}{ Mali (2006) } \\
\hline & Effectif & $\begin{array}{l}\% \text { en retard de } \\
\text { vaccination }\end{array}$ & Effectif & $\begin{array}{l}\% \text { en retard de } \\
\text { vaccination }\end{array}$ \\
\hline Plateau Central & 149 & $71,8 \%$ & & - \\
\hline Sud-Ouest & 118 & $78,8 \%$ & & - \\
\hline Niveau de vie contextuel & & ns & & $* * *$ \\
\hline$<$ Médiane & 558 & $76,8 \%$ & 1090 & $81,7 \%$ \\
\hline$\geq$ Médiane & 558 & $74,1 \%$ & 1090 & $59,6 \%$ \\
\hline Niveau d'instruction contextuel & & ns & & **** \\
\hline$<$ Médiane & 558 & $77,6 \%$ & 1090 & $75,8 \%$ \\
\hline$\geq$ Médiane & 558 & $73,4 \%$ & 1090 & $65,8 \%$ \\
\hline Ensemble & 3116 & $75,5 \%$ & 2180 & $71,0 \%$ \\
\hline
\end{tabular}

\section{Analyse multiniveau}

Les modèles d'analyse confirment immanquablement l'influence du contexte sur le retard dans la vaccination des enfants, quel que soit le pays. En effet, tous les effets aléatoires apparaissent comme étant significatifs $(p<0,1 \%)$. Le coefficient de partition de la variance indique également qu'une partie de la propension des enfants à recevoir leurs vaccins en retard est attribuable aux différences contextuelles. Cependant, après contrôle par les facteurs individuels, les seuls facteurs contextuels significatifs ont été la région (au Burkina Faso et au Mali) et le niveau de vie moyen de la zone de dénombrement (au Mali) (Tableau 5). Dans le modèle final, trois facteurs sont significativement déterminants dans l'explication du retard de vaccination simultanément au Mali et au Burkina Faso (cf. Tableau 5). II s'agit du lieu de naissance de l'enfant, de la région et du niveau d'instruction de la mère.

Au niveau individuel, un plus grand nombre de facteurs ressortent comme étant significativement associés au retard dans la vaccination des enfants au Burkina Faso, par rapport au Mali. (cf. Tableau 5). Au niveau de l'enfant, il s'agit du rang de naissance et de l'âge de la mère à la naissance de l'enfant. Au niveau de la mère, il s'agit de la religion, du niveau de vie du ménage et du statut matrimonial de la mère. Ainsi, le modèle final indique que les enfants burkinabé de mères mariées ont plus de risque de recevoir leurs vaccins en retard aux âges spécifiques que ceux de mères hors mariage, toutes choses égales par ailleurs.

On notera également que le sexe de l'enfant est le seul facteur relatif à l'enfant qui s'avère significatif au Mali mais pas au Burkina Faso (cf. Tableau 5). Les garçons maliens ont $I, 25$ fois plus de risque $(p<10 \%)$ d'être vaccinés en retard aux âges spécifiques que les filles maliennes, toutes choses égales par ailleurs. Au niveau contextuel, le seul facteur qui est significatif au
Mali sans l'être au Burkina Faso est le niveau de vie contextuel. L'analyse de ce facteur indique qu'une augmentation d'un point du score factoriel moyen de la $Z D$ réduit de $3 \%(p<10 \%)$ le risque pour un enfant malien de recevoir un vaccin en retard (toutes choses égales par ailleurs).

Quel que soit que le pays, la région de résidence au moment de l'enquête est associée à des variations significatives. Ainsi, les enfants des régions du CentreEst, de l'Ouest, du Sud et du Plateau courent un risque moindre de recevoir leurs vaccins en retard, par rapport à ceux du Centre au Burkina Faso. Au Mali, comparativement aux enfants de Bamako, ceux de la région de Ségou ont un risque qui $46 \%$ $(\mathrm{p}<10 \%)$ inférieur, tandis que ceux de la région de Koulikoro ont I,64 ( $p<5 \%)$ fois plus de risque d'être vaccinés en retard.

La significativité des variances contextuelles suggère qu'il existe d'autres facteurs contextuels non pris en compte dans les modèles et qui influencent le risque individuel de retard de vaccination. Une investigation plus approfondie, par exemple à l'aide d'une approche qualitative axée autant sur le contexte que sur les individus pourrait aider à compléter l'explication du retard de vaccination. Cette significative indique également que les chances de recevoir les vaccins à temps varient sensiblement d'un contexte à un autre. Ceci constitue un argument supplémentaire justifie l'utilisation de l'approche multiniveau pour expliquer le retard de vaccination.

La contribution des différences contextuelles est estimée à 28,2\% au Mali et 13,4\% au Burkina Faso dans le modèle M0. Ceci confirme qu'une importante partie de l'explication du retard de vaccination reste attribuable aux différences individuelles, ce qui confirme globalement notre seconde hypothèse $(\mathrm{H} 2)$. On relèvera toutefois que le Burkina Faso semble mieux s'être affranchi des contraintes communautaires que le Mali. 
Tableau 5 : Rapport de chances du retard dans la vaccination des enfants aux âges spécifiques au Burkina Faso et au Mali

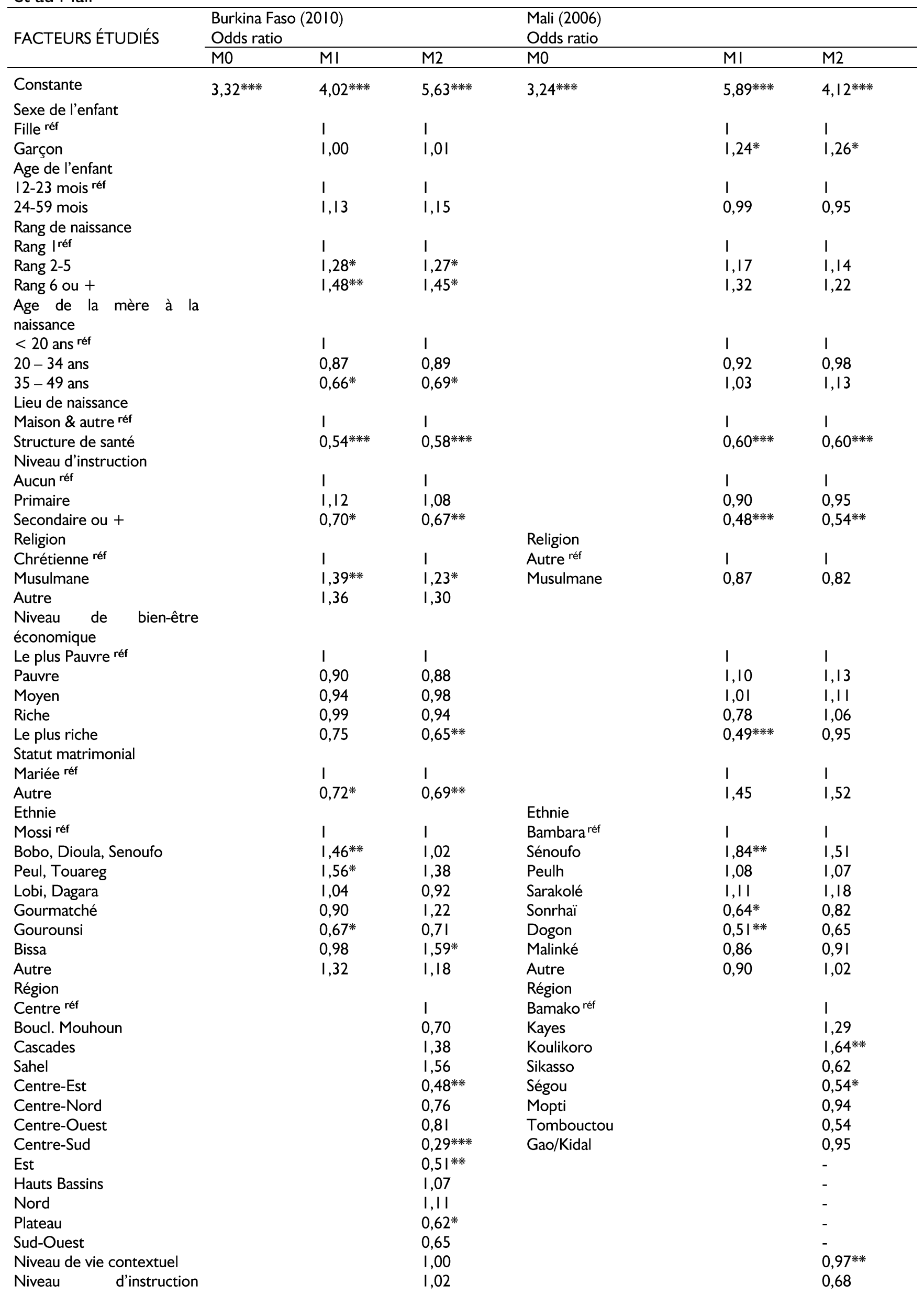




\begin{tabular}{|c|c|c|c|c|c|c|}
\hline \multirow{2}{*}{ FACTEURS ÉTUDIÉS } & \multicolumn{3}{|c|}{$\begin{array}{l}\text { Burkina Faso }(20 \mid 0) \\
\text { Odds ratio }\end{array}$} & \multicolumn{3}{|l|}{$\begin{array}{l}\text { Mali (2006) } \\
\text { Odds ratio }\end{array}$} \\
\hline & MO & $\mathrm{MI}$ & $M 2$ & Mo & MI & $M 2$ \\
\hline \multicolumn{7}{|l|}{$\begin{array}{l}\text { contextuel } \\
\text { VARIANCE }\end{array}$} \\
\hline Contextuelle & 0,5 I**** & $0,41 * * *$ & $0,28 * * *$ & $\mathrm{I}, 29 * * * *$ & 0,73 **** & $0,69 * * * *$ \\
\hline CPV & $13,4 \%$ & $11,3 \%$ & $7,8 \%$ & $28,2 \%$ & $18,1 \%$ & $17,3 \%$ \\
\hline
\end{tabular}

Significativité : $* \mathrm{p} \leq 10 \%, \quad * * \mathrm{p} \leq 5 \%, * * * \mathrm{p} \leq 0,1 \%, \mathrm{CPV}=$ Coefficient de partition de la variance

\section{Discussion}

Plusieurs études ont porté sur les caractéristiques associées au retard dans la vaccination des enfants, mais peu d'entre elles ont mis l'accent sur l'influence du contexte socioéconomique du voisinage.

Notre étude met en exergue la fréquence du retard aux âges de vaccination au Burkina Faso et au Mali et évalue le rôle des déterminants contextuels dans son explication. Dans les deux pays, le retard à la vaccination concerne un peu plus de sept enfants sur dix. Certains facteurs socioéconomiques y sont significativement associés, tels que le sexe, le rang de naissance, le lieu de naissance et l'âge de la mère à la naissance de l'enfant. Le niveau d'instruction de la mère, son statut matrimonial, sa religion et le niveau de vie de son ménage sont également associés à des inégalités en matière de respect du calendrier, tout comme l'est la région et le niveau de vie contextuel.

Les modèles d'analyse multiniveau confirment l'influence du contexte sur le retard dans la vaccination des enfants, et ce, dans les deux pays. Cependant, les seuls facteurs contextuels qui s'avèrent significatifs après contrôle par les facteurs individuels sont la région (au Burkina Faso et au Mali) et le niveau de vie moyen de la zone de dénombrement (au Mali). Nos résultats indiquent donc que les déterminants individuels de la santé exercent une plus forte influence que ceux relatifs au contexte dans l'explication du retard de vaccination chez les enfants. L'analyse du coefficient de partition de la variance confirme qu'une part importante de l'explication du retard de vaccination reste logée au niveau individuel dans les deux pays (ce que DiezRoux et Mair (20I0) ainsi que Galster (20I0) avaient déjà pu montrer).

De façon spécifique, il ressort que l'état matrimonial de la mère (au Burkina Faso) et le sexe de l'enfant sont significativement associés au retard vaccinal, mais dans un sens inattendu par rapport à la littérature (cf. Tableau 5). En effet, les garçons maliens présentent plus de risque de recevoir leurs vaccins en retard que les filles. Or les garçons sont généralement favorisés dans les sociétés africaines, et particulièrement dans la société malienne (Dombkowski et al., 2004 ; MBacké et Legrand,
1992). Deux phénomènes peuvent expliquer le résultat contre-intuitif observé ici; (I) un biais de sélection (prise en compte uniquement des enfants disposant d'un carnet) et surtout le seuil élevé de significativité $(10 \%)$ auquel ce résultat est considéré comme significatif.

De façon plus attendue, l'âge de l'enfant, son rang de naissance, son lieu de naissance, ainsi que , l'âge de la mère, son niveau d'instruction, sa religion, son statut matrimonial et le niveau de vie du ménage sont tous significativement associés au retard dans la vaccination des enfants. Par contre, le groupe ethnique et le niveau d'instruction contextuel ne présentent pas de relation significative avec le retard de vaccination. Dans le même ordre d'idée, Soura et al. (20/3) avaient déjà montré que le groupe ethnique de la mère n'avait pas d'influence sur le statut vaccinal de l'enfant à Ouagadougou.

Un résultat marquant de notre recherche porte sur le nombre élevé de facteurs significativement associés au retard de vaccination au Burkina Faso, comparativement au Mali. Ceci laisse penser que les politiques et programmes de vaccination du Burkina Faso effaceraient moins les inégalités entre les couches socioéconomiques (niveau de vie, ethnie, statut matrimonial, etc.) qu'au Mali, bien que la couverture vaccinale soit plus élevée au Burkina Faso. $\mathrm{Ce}$ résultat invite donc les décideurs burkinabé à repenser les programmes de vaccination qui semblent ne pas profiter de façon équitable à l'ensemble des couches de la population.

Enfin, sur un plan méthodologique, précisons que la définition du retard vaccinal peut changer d'un auteur à un autre et influencer les résultats de recherche. II n'y a pour le moment pas de mesure unanimement admise du retard de vaccination chez les enfants. Le délai de quatre semaine retenu par l'étude (et dans plusieurs travaux consultés) permet d'éviter un chevauchement entre les tranches d'âges acceptables pour l'administration des vaccinations aux enfants. Les résultats sont toutefois fortement dépendants de la mesure proposée. II conviendrait que les chercheurs et les professionnels de santé adoptent une définition et une mesure qui puissent permettre des comparaisons robustes dans le temps et dans l'espace. 
Avant de conclure, rappelons que cette étude n'est pas exempte d'insuffisances relatives non seulement à l'échantillon mais également aux facteurs pris en compte dans les modèles. Une première limite porte sur les biais de sélection des individus lors des enquêtes mais surtout l'échantillon ayant servi à estimer les modèles d'analyses. En effet, les données analysées sont relatives aux enfants en vie et disposant de carnet de vaccination. Les informations relatives à ceux décédés ou à ceux ne disposant pas de carnet de vaccination pourraient s'avérer utiles pour mieux apprécier le respect du calendrier vaccinal à l'échelle du pays. La seconde limite concerne la non prise en compte de certains facteurs tels que les variables de nature psychologique relatives aux idées que les parents se font de l'efficacité et de la pertinence des vaccins administrés aux enfants (Smith et al., 20l0). Au niveau communautaire, la proximité à une formation sanitaire est une variable qui devrait être intégrée dans de futures analyses. La troisième limite concerne le décalage constaté sur les dates de collecte des données au Burkina Faso (2010) et au Mali (2006). Ce décalage entre les dates de collecte ne permet pas stricto sensu une analyse comparative des effets des programmes respectifs de vaccination de ces pays. Enfin, nous avons considéré ici le retard sur l'ensemble des vaccins, et il semble utile dans le futur d'analyser le retard par type de vaccin.

\section{Conclusion}

Cette recherche a rappelé la fréquence du retard dans la vaccination des enfants au Mali et au Burkina Faso (qui touche un peu plus de sept enfants sur dix). Ce travail a également confirmé les hypothèses selon lesquelles le voisinage influence effectivement le respect du calendrier vaccinal de l'enfant, bien que ce respect reste davantage tributaire des caractéristiques individuelles de l'enfant et de celles relatives à sa mère. La région et le niveau de vie du voisinage ont été identifiés comme des facteurs contextuels pouvant entraver le respect du calendrier vaccinal de l'enfant. Par ailleurs, l'étude a permis de mettre en exergue l'existence d'inégalités face à la vaccination plus prononcées au Burkina Faso qu'au Mali. Par contre, le contexte semble avoir une influence plus nette au Mali sur la vaccination des enfants.

Enfin, un nombre plus important de facteurs individuels s'avèrent significatifs au Burkina Faso, tandis que le niveau de vie contextuel est apparu significatif au Mali, mais pas au Burkina Faso. Bien que les deux pays considérés soient géographiquement et socio-économiquement proches, notre étude pointe donc vers des influences communautaires différentes qui méritent des approfondissements.

\section{Remerciements}

Les auteurs remercient le Centre de Recherche pour le Développement International (CRDI) pour avoir financé la formation de l'un des co-auteurs. Egalement, ils sont redevables au Programme DHS de ICF International qui a autorisé l'utilisation des données EDS.

\section{Références}

Akoto, M. E., et Kandem, H. K. (200I). Etude comparative des déterminants de la pratique contraceptive moderne en Afrique. In F. Gendreau (Ed.), Transitions Démographiques des pays du Sud (ESTEM., pp. 269-286). Yaoundé.

Babalola, S. (2008). Determinants of the uptake of the full dose of diphtheria-pertussis-tetanus vaccines (DPT3) in Northern Nigeria: a multilevel analysis. Maternal and Child Health Journal, I3(4), 550-558.

Cellule de Planification et de Statistique Ministère de la Santé, Direction Nationale de la Statistique et de l'Informatique du Ministère de l'Économie de I'Industrie et du Commerce (DNSI/MEIC), et Macro International Inc. (2007). Enquête Démographique et de Santé du Mali 2006 (p. 535). Calverton, Maryland, USA: CPS/DNSI et Macro International Inc.

Clark, A., et Sanderson, C. (2009). Timing of children's vaccinations in 45 low-income and middle-income countries: an analysis of survey data. The Lancet, 373(9674), I543-1549.

Diez-Roux, A. V, et Mair, C. (2010). Neighborhoods and health. Annals of the New York Academy of Sciences, I/86, I25-I45.

Dombkowski, K. J., Lantz, P. M., et Freed, G. L. (2004). Risk factors for delay in age-appropriate vaccination. Public Health Reports, I/9(2), I44155.

Galster, G. C. (20|0). The Mechanism (s) of Neighborhood Effects Theory, Evidence, and Policy Implications. In ESRC Seminar: "Neighbourhood Effects: Theory et Evidence" (pp. I-32). St. Andrews University, Scotland, UK.

INSTAT. (20II). 4eme Recensement General de la Population et de l'Habitat du Mali (RGPH-2009): Analyse des Résultats Définitifs "Thème 2: Etat et Structure de la Population». (Institut National de la Statistique, Ed.) (p. I 19). Bamako.

Institut National de la Statistique et de la Démographie (INSD), et ICF International. (2012). Enquête Démographique et de Santé (EDS-IV) et à Indicateurs Multiples (MICS) du 
Burkina Faso (p. 527). Calverton, Maryland, USA: ICF International et INSD.

Leckie, G., et Charlton, C. (20/2). runmlwin: A Program to Run the MLwiN Multilevel Modeling Software from within Stata. Journal of Statistical Software, 52(I I), I-40.

Masquelier, B.; Reniers, G. et Pison, G. (20/4). Divergences in mortality trends in sub-Saharan Africa: survey evidence on the survival of children and siblings Population Studies, 68, I6I-I77

MBacké, C. S. M., et Legrand, T. K. (1992). Différence de mortalité selon le sexe et utilisation des services de santé au Mali. Cahiers Québécois de Démographie, 2I(I), 99-II9.

Papania, M., et Rodewald, L. (2006). For better immunisation coverage, measure coverage better. The Lancet, 367, 965-966.

Pickett, K. E., et Pearl, M. (200I). Multilevel analyses of neighbourhood socioeconomic context and health outcomes: a critical review. J Epidemiol Community Health, 55, I I I-I 22.

PNUD. (2013). Rapport sur le développement humain 2013: L'essor du Sud - Le progrès humain dans un monde divrsifié. (W. D. Communications Development Incorporated, Ed.) (p. 228). New York: Communications Development Incorporated, Washington DC.

Saliou, P. (2007). Vaccination et développement en Afrique sub-saharienne. Bulletin de l'Académie Nationale de Médecine, 19I(8), 1589-1600.
Sia, D., Kobiané, J., et Fournier, P. (2007). Étude originale Les facteurs individuels et du milieu de vie associés à la vaccination complète des enfants en milieu rural au Burkina Faso: une approche multiniveau. Cahiers Santé, I 7(4), 20 I-206.

Smith, P. J., Humiston, S. G., Parnell, T., Vannice, K. S., Salmon, A., et Salmon, D. A. (20I0). The Association Between Intentional Delay of Vaccine Administration and Timely Childhood Vaccination Coverage. Public Health Reports, I25(4), 534$54 \mathrm{I}$.

Soura, A., Pison, G., Senderowicz, L., et Rossier, C. (20/3). Religious differences in child vaccination rates in urban Africa: Comparison of population surveillance data from Ouagadougou , Burkina Faso. African Population Studies, 27(2), 74-87.

Turrell, G. (1995). Reducing Socioeconomic Health Inequalities: Issues of Relevance for Policy. NSW Public Health Bulletin, 13(3), 47-49.

Wiysonge, C. S., Uthman, O. a, Ndumbe, P. M., et Hussey, G. D. (20|2). Individual and contextual factors associated with low childhood immunisation coverage in sub-Saharan Africa: a multilevel analysis. PloS One, 7(5), e37905.

Wiysonge, C. S., Uthman, O. a, Ndumbe, P. M., et Hussey, G. D. (20/3). A bibliometric analysis of childhood immunization research productivity in Africa since the onset of the Expanded Program on Immunization in 1974. BMC Medicine, I I, 66. 